\title{
BUCHSBAUMNESS IN LOCAL RINGS POSSESSING CONSTANT FIRST HILBERT COEFFICIENTS OF PARAMETERS
}

\author{
SHIRO GOTO AND KAZUHO OZEKI
}

\begin{abstract}
Let $(A, \mathfrak{m})$ be a Noetherian local ring with $d=\operatorname{dim} A \geq 2$. Then, if $A$ is a Buchsbaum ring, the first Hilbert coefficients $\mathrm{e}_{Q}^{1}(A)$ of $A$ for parameter ideals $Q$ are constant and equal to $-\sum_{i=1}^{d-1}\left(\begin{array}{c}d-2 \\ i-1\end{array}\right) h^{i}(A)$, where $h^{i}(A)$ denotes the length of the $i$ th local cohomology module $\mathrm{H}_{\mathfrak{m}}^{i}(A)$ of $A$ with respect to the maximal ideal $\mathfrak{m}$. This paper studies the question of whether the converse of the assertion holds true, and proves that $A$ is a Buchsbaum ring if $A$ is unmixed and the values $\mathrm{e}_{Q}^{1}(A)$ are constant, which are independent of the choice of parameter ideals $Q$ in $A$. Hence, a conjecture raised by [GhGHOPV] is settled affirmatively.
\end{abstract}

\section{$\S 1$. Introduction}

The purpose of this paper is to affirmatively answer a conjecture raised in [GhGHOPV], which claims Buchsbaumness in local rings possessing constant first Hilbert coefficients of parameter ideals.

To explicitly state the conjecture and our results, let us fix some notation. In this article, let $A$ be a Noetherian local ring with maximal ideal $\mathfrak{m}$, and let $d=\operatorname{dim} A>0$. Let $\ell_{A}(M)$ denote, for an $A$-module $M$, the length of $M$. Then for each m-primary ideal $I$ in $A$, we have integers $\left\{\mathrm{e}_{I}^{i}(A)\right\}_{0 \leq i \leq d}$ such that the equality

$$
\ell_{A}\left(A / I^{n+1}\right)=\mathrm{e}_{I}^{0}(A)\left(\begin{array}{c}
n+d \\
d
\end{array}\right)-\mathrm{e}_{I}^{1}(A)\left(\begin{array}{c}
n+d-1 \\
d-1
\end{array}\right)+\cdots+(-1)^{d} \mathrm{e}_{I}^{d}(A)
$$

holds true for all $n \gg 0$, which we call the Hilbert coefficients of $A$ with respect to $I$. We say that $A$ is unmixed if $\operatorname{dim} \widehat{A} / \mathfrak{p}=d$ for every $\mathfrak{p} \in$ Ass $\widehat{A}$, where $\widehat{A}$ denotes the $\mathfrak{m}$-adic completion of $A$. Let $\mathrm{H}_{\mathfrak{m}}^{i}(*)(i \in \mathbb{Z})$ denote the local cohomology functors of $A$ with respect to $\mathfrak{m}$.

Received April 28, 2009. Revised November 2, 2009. Accepted November 26, 2009.

2000 Mathematics Subject Classification. Primary 13H10; Secondary 13A30, 13B22, $13 \mathrm{H} 15$.

(C) 2010 by The Editorial Board of the Nagoya Mathematical Journal 
With this notation, the main result of this paper is the following.

Theorem 1.1. Suppose that $d=\operatorname{dim} A \geq 2$ and that $A$ is unmixed. Then the following two conditions are equivalent.

(1) A is a Buchsbaum local ring.

(2) The first Hilbert coefficients $\mathrm{e}_{Q}^{1}(A)$ of $A$ are constant and independent of the choice of parameter ideals $Q$ in $A$.

When this is the case, one has the equality

$$
\mathrm{e}_{Q}^{1}(A)=-\sum_{i=1}^{d-1}\left(\begin{array}{l}
d-2 \\
i-1
\end{array}\right) \ell_{A}\left(\mathrm{H}_{\mathfrak{m}}^{i}(A)\right)
$$

for every parameter ideal $Q$ in $A$.

Here let us briefly recall the definition of Buchsbaum local rings. The readers may consult the monumental book by Stückrad and Vogel [SV] for a detailed theory, some of which we note here for use in this paper.

We say that our local ring $A$ is Buchsbaum if the difference

$$
\ell_{A}(A / Q)-\mathrm{e}_{Q}^{0}(A)
$$

is independent of the choice of parameter ideals $Q$ in $A$ and is an invariant of $A$, which we denote by $\mathbb{I}(A)$. As is well known, $A$ is a Buchsbaum ring if and only if every system $a_{1}, a_{2}, \ldots, a_{d}$ of parameters in $A$ forms a $d$-sequence in the sense of Huneke $[\mathrm{H}]$. When $A$ is a Buchsbaum local ring, one has

$$
\mathfrak{m} \cdot \mathrm{H}_{\mathfrak{m}}^{i}(A)=(0)
$$

for all $i \neq d$. Therefore the local cohomology modules $\left\{\mathrm{H}_{\mathfrak{m}}^{i}(A)\right\}_{i \neq d}$ are finitedimensional vector spaces over the field $A / \mathfrak{m}$, and the equality

$$
\mathbb{I}(A)=\sum_{i=0}^{d-1}\left(\begin{array}{c}
d-1 \\
i
\end{array}\right) \ell_{A}\left(\mathrm{H}_{\mathfrak{m}}^{i}(A)\right)
$$

holds true.

We say that $A$ is a generalized Cohen-Macaulay local ring if all the local cohomology modules $\left\{\mathrm{H}_{\mathfrak{m}}^{i}(A)\right\}_{i \neq d}$ are finitely generated. Hence, every Cohen-Macaulay local ring is Buchsbaum with $\mathbb{I}(A)=0$, and Buchsbaum local rings are generalized Cohen-Macaulay. A given Noetherian local ring $A$ with $d=\operatorname{dim} A>0$ is a generalized Cohen-Macaulay local ring if and only if

$$
\mathbb{I}(A):=\sup _{Q}\left\{\ell_{A}(A / Q)-\mathrm{e}_{Q}^{0}(A)\right\}<\infty
$$


where $Q$ runs through parameter ideals in $A[\mathrm{STC}]$. When this is the case, one has

$$
\mathbb{I}(A)=\sum_{i=0}^{d-1}\left(\begin{array}{c}
d-1 \\
i
\end{array}\right) \ell_{A}\left(\mathrm{H}_{\mathfrak{m}}^{i}(A)\right) .
$$

Suppose that $A$ is a generalized Cohen-Macaulay local ring, and let $Q$ be a parameter ideal in $A$. Then $Q$ is called standard if

$$
\mathbb{I}(A)=\ell_{A}(A / Q)-\mathrm{e}_{Q}^{0}(A) .
$$

This condition is equivalent to saying that $Q$ is generated by a system $a_{1}, a_{2}, \ldots, a_{d}$ of parameters which forms a strong $d$-sequence in any order [STC].

Theorem 1.1 asserts that Buchsbaum local rings $A$ with $\operatorname{depth} A>0$ are characterized by the consistency of $\mathrm{e}_{Q}^{1}(A)$ for parameter ideals $Q$. Notice that in Theorem 1.1 our contribution is the implication $(2) \Rightarrow(1)$; the implication $(1) \Rightarrow(2)$ and the last assertion are due to Schenzel [Sch, Korollar 3.2].

As a consequence of Theorem 1.1, we also have a characterization of local rings $A$ which are not necessarily unmixed to possess constant first Hilbert coefficients $\mathrm{e}_{Q}^{1}(A)$ for parameter ideals $Q$ (see Theorem 3.2). However, before entering more details, let us explain the background of our research.

Our research dates back to the following conjecture of Vasconcelos posed at the conference in Yokohama of March 2008 [V].

Conjecture $1.2[\mathrm{~V}]$. Assume that $A$ is unmixed. Then $A$ is a CohenMacaulay local ring once $\mathrm{e}_{Q}^{1}(A)=0$ for some parameter ideal $Q$ of $A$.

This conjecture was partially solved by [GhHV] and recently settled affirmatively by [GhGHOPV, Theorems 2.1, 3.4]. Mandal and Verma ([MV, Theorem 8]; see also [GhGHOPV, Corollary 2.5]) showed that $\mathrm{e}_{Q}^{1}(A) \leq 0$ for every parameter ideal $Q$ in arbitrary Noetherian local rings $A$ with $\operatorname{dim} A>0$. The authors of [GhGHOPV] thereafter started the study of the next extreme case, that is, the case where the set

$$
\Lambda(A)=\left\{\mathrm{e}_{Q}^{1}(A) \mid Q \text { is a parameter ideal in } A\right\}
$$

is finite. Among their results, one finds that $A$ is a generalized CohenMacaulay local ring, once the set $\Lambda(A)$ is finite and $A$ is unmixed 
([GhGHOPV, Proposition 4.2]). Because $\mathrm{e}_{Q}^{1}(A)$ is constant if $A$ is a Buchsbaum local ring (see [Sch, Korollar 3.2]), it seems now very natural to conjecture that $A$ is a Buchsbaum local ring if $A$ is unmixed and the value $\mathrm{e}_{Q}^{1}(A)$ is constant, which our Theorem 1.1 settles affirmatively. (See [GhGHOPV, Theorems 4.8, 4.10] for partial answers for the cases where $\Lambda(A)=\{-1\}$ and $\Lambda(A)=\{-2\}$, respectively.)

We now explain how this paper is organized. Theorem 1.1 is proved in Section 2. When $A$ is a generalized Cohen-Macaulay local ring with $d=$ $\operatorname{dim} A \geq 2$, one has

$$
0 \geq \mathrm{e}_{Q}^{1}(A) \geq-\sum_{i=1}^{d-1}\left(\begin{array}{l}
d-2 \\
i-1
\end{array}\right) \ell_{A}\left(\mathrm{H}_{\mathfrak{m}}^{i}(A)\right)
$$

for every parameter ideal $Q$ in $A$ ([MV, Theorem 8], [GNi, Lemma 2.4]), where the equality

$$
\mathrm{e}_{Q}^{1}(A)=-\sum_{i=1}^{d-1}\left(\begin{array}{l}
d-2 \\
i-1
\end{array}\right) \ell_{A}\left(\mathrm{H}_{\mathfrak{m}}^{i}(A)\right)
$$

holds for standard parameter ideals $Q$ ([Sch, Korollar 3.2]); hence, the set $\Lambda(A)$ is finite. Because $A$ is a Buchsbaum local ring if and only if $A$ is a generalized Cohen-Macaulay local ring in which every parameter ideal is standard, the heart of our proof of Theorem 1.1 is to show that $Q$ is standard, once $\mathrm{e}_{Q}^{1}(A)=-\sum_{i=1}^{d-1}\left(\begin{array}{c}d-2 \\ i-1\end{array}\right) \ell_{A}\left(\mathrm{H}_{\mathfrak{m}}^{i}(A)\right)$, assuming $A$ is a generalized Cohen-Macaulay local ring with $d=\operatorname{dim} A \geq 2$ and $\operatorname{depth} A>0$. We perform this task in Section 2 (Theorem 2.1). The implication $(2) \Rightarrow(1)$ is no longer true unless $A$ is unmixed, which we show by Examples 2.5 and 2.6.

In Section 3, following the steps developed in [GhGHOPV], we give a characterization for general local rings to have $\mathrm{e}_{Q}^{1}(A)$ constant for all parameter ideals $Q$ in $A$ (Theorem 3.2).

\section{§2. Proof of Theorem 1.1}

Let $A$ be a Noetherian local ring with maximal ideal $\mathfrak{m}$ and $d=\operatorname{dim} A>0$. Let $a_{1}, a_{2}, \ldots, a_{d}$ be a system of parameters of $A$. Then $a_{1}, a_{2}, \ldots, a_{d}$ is said to be a standard system of parameters if it forms a $d^{+}$-sequence, that is, if $a_{1}, a_{2}, \ldots, a_{d}$ forms a strong $d$-sequence in any order. This condition is equivalent to saying that $A$ is a generalized Cohen-Macaulay local ring and that

$$
\mathbb{I}(A)=\ell_{A}(A / Q)-\mathrm{e}_{Q}^{0}(A)
$$


([T, Theorem 2.1]), where $Q=\left(a_{1}, a_{2}, \ldots, a_{d}\right)$. We simply say that a parameter ideal $Q$ of $A$ is standard if it is generated by a standard system of parameters. When $A$ is a generalized Cohen-Macaulay local ring, one can choose an integer $\ell \gg 0$ so that every system of parameters contained in $\mathfrak{m}^{\ell}$ is standard ([STC, Satz 3.7]). (See [STC] and [T] for details, where the notion of generalized Cohen-Macaulay module is also given and various important properties of generalized Cohen-Macaulay local rings and modules are explored.)

We put $h^{i}(A)=\ell_{A}\left(\mathrm{H}_{\mathfrak{m}}^{i}(A)\right)$ for each $i \in \mathbb{Z}$. The following result is the heart of this paper.

Theorem 2.1. Suppose that $A$ is a generalized Cohen-Macaulay local ring with $d=\operatorname{dim} A \geq 2$ and $\operatorname{depth} A>0$. Let $Q$ be a parameter ideal in $A$. Then the following two conditions are equivalent.

(1) $Q$ is a standard parameter ideal in $A$.

(2) $\mathrm{e}_{Q}^{1}(A)=-\sum_{i=1}^{d-1}\left(\begin{array}{c}d-2 \\ i-1\end{array}\right) h^{i}(A)$.

To prove Theorem 2.1, we need the following.

Lemma 2.2 ([GhGHOPV, Lemma 4.5]). Suppose that $A$ is a generalized Cohen-Macaulay local ring with $d=\operatorname{dim} A \geq 2$ and $\operatorname{depth} A>0$. Let $Q$ be a parameter ideal in $A$, and assume that $\mathrm{e}_{Q}^{1}(A)=-\sum_{i=1}^{d-1}\left(\begin{array}{c}d-2 \\ i-1\end{array}\right) h^{i}(A)$. Then $Q \cdot \mathrm{H}_{\mathfrak{m}}^{i}(A)=(0)$ for all $1 \leq i \leq d-1$.

For each ideal $\mathfrak{a}$ in $A(\mathfrak{a} \neq A)$, let $\mathrm{U}(\mathfrak{a})$ denote the unmixed component of $\mathfrak{a}$. When $\mathfrak{a}=(a)$ with $a \in A$, we write $\mathrm{U}(\mathfrak{a})$ simply by $\mathrm{U}(a)$. We have

$$
\mathrm{U}(a)=\bigcup_{n \geq 0}\left[(a): A_{A} \mathfrak{m}^{n}\right],
$$

if $A$ is a generalized Cohen-Macaulay local $\operatorname{ring}$ with $\operatorname{dim} A \geq 2$ and $a$ is a part of a system of parameters in $A$ (see [STC, Section 2]).

The following result is the key in our proof of Theorem 2.1.

Proposition 2.3. Suppose that $A$ is a generalized Cohen-Macaulay local ring with $d=\operatorname{dim} A \geq 3$ and depth $A>0$. Let $Q=\left(a_{1}, a_{2}, \ldots, a_{d}\right)$ be a parameter ideal in $A$. Assume that $\left(a_{1}, a_{d}\right) \mathrm{H}_{\mathfrak{m}}^{1}(A)=(0)$ and that the parameter ideal $\left(a_{1}, a_{2}, \ldots, a_{d-1}\right) \cdot\left[A / \mathrm{U}\left(a_{d}\right)\right]$ is standard in the generalized CohenMacaulay local ring $A / \mathrm{U}\left(a_{d}\right)$. Then

$$
\mathrm{U}\left(a_{1}\right) \cap Q=\left(a_{1}\right) .
$$


Proof. Since $\mathrm{U}\left(a_{1}\right) \cap Q=\left(a_{1}\right)+\left[\mathrm{U}\left(a_{1}\right) \cap\left(a_{2}, a_{3}, \ldots, a_{d}\right)\right]$, we have only to show that

$$
\mathrm{U}\left(a_{1}\right) \cap\left(a_{2}, a_{3}, \ldots, a_{d}\right) \subseteq\left(a_{1}\right)
$$

Let $x \in \mathrm{U}\left(a_{1}\right) \cap\left(a_{2}, a_{3}, \ldots, a_{d}\right)$, and put $\bar{A}=A / \mathrm{U}\left(a_{d}\right)$. Let $\bar{x}$ and $\overline{a_{i}}$, respectively, denote the images of $x$ and $a_{i}$ in $\bar{A}$. Then we have

$$
\bar{x} \in \mathrm{U}\left(\overline{a_{1}}\right) \cap\left(\overline{a_{2}}, \overline{a_{3}}, \ldots, \overline{a_{d-1}}\right) \subseteq\left(\overline{a_{1}}\right),
$$

because $\mathrm{U}\left(\overline{a_{1}}\right)=\left(\overline{a_{1}}\right): \bar{A} \overline{a_{2}}$ and $\overline{a_{2}}, \overline{a_{3}}, \ldots, \overline{a_{d-1}}$ forms a $d$-sequence in $\bar{A}$. (Recall that, by our assumption, $\left(\overline{a_{1}}, \overline{a_{2}}, \ldots, \overline{a_{d-1}}\right)$ is a standard parameter ideal in $\bar{A}$.) Hence,

$$
x \in\left[\left(a_{1}\right)+\mathrm{U}\left(a_{d}\right)\right] \cap \mathrm{U}\left(a_{1}\right)=\left(a_{1}\right)+\left[\mathrm{U}\left(a_{1}\right) \cap \mathrm{U}\left(a_{d}\right)\right] .
$$

Let $x=y+z$ with $y \in\left(a_{1}\right)$ and $z \in \mathrm{U}\left(a_{1}\right) \cap \mathrm{U}\left(a_{d}\right)$. We will show that $z \in\left(a_{1}\right)$.

Since $a_{1} \mathrm{H}_{\mathfrak{m}}^{1}(A)=(0)$ and $a_{1}$ is $A$-regular, we have

$$
\mathrm{H}_{\mathfrak{m}}^{1}(A) \cong \mathrm{H}_{\mathfrak{m}}^{0}\left(A /\left(a_{1}\right)\right)=\mathrm{U}\left(a_{1}\right) /\left(a_{1}\right) .
$$

Therefore $a_{d} \mathrm{U}\left(a_{1}\right) \subseteq\left(a_{1}\right)$, because $a_{d} \mathrm{H}_{\mathfrak{m}}^{1}(A)=(0)$ by our assumption. By the same argument applied to $a_{d}$, we get $a_{1} \mathrm{U}\left(a_{d}\right) \subseteq\left(a_{d}\right)$. Hence, $a_{1} z \in\left(a_{d}\right)$ and $a_{d} z \in\left(a_{1}\right)$. Let us now write

$$
a_{1} z=a_{d} u \quad \text { and } \quad a_{d} z=a_{1} v, \quad \text { with } u, v \in A \text {. }
$$

Then, since $a_{1} a_{d} z=a_{d}^{2} u=a_{1}^{2} v$, we have $u \in \mathrm{U}\left(a_{1}^{2}\right)$. Notice that

$$
\mathrm{H}_{\mathfrak{m}}^{1}(A) \cong \mathrm{H}_{\mathfrak{m}}^{0}\left(A /\left(a_{1}^{2}\right)\right)=\mathrm{U}\left(a_{1}^{2}\right) /\left(a_{1}^{2}\right),
$$

since $a_{1}^{2} \mathrm{H}_{\mathfrak{m}}^{1}(A)=(0)$ and $a_{1}^{2}$ is $A$-regular. Therefore, $a_{d} \mathrm{U}\left(a_{1}^{2}\right) \subseteq\left(a_{1}^{2}\right)$, because $a_{d} \mathrm{H}_{\mathfrak{m}}^{1}(A)=(0)$. Hence, $a_{1} a_{d} z=a_{d} \cdot a_{d} u \in\left(a_{1}^{2} a_{d}\right)$, so that $z \in\left(a_{1}\right)$. Thus $x=y+z \in\left(a_{1}\right)$, as is claimed.

We are now in a position to prove Theorem 2.1 .

Proof of Theorem 2.1. It suffices to show the implication $(2) \Rightarrow(1)$. Enlarging the residue class field $A / \mathfrak{m}$ of $A$ if necessary, we may assume that the field $A / \mathfrak{m}$ is infinite. Let $Q=\left(a_{1}, a_{2}, \ldots, a_{d}\right)$, where each $a_{j}$ is superficial for the ideal $Q$. Recall that $Q \mathrm{H}_{\mathfrak{m}}^{i}(A)=(0)$ for all $1 \leq i \leq d-1$ by Lemma 2.2 . Hence, $Q$ is standard, if $d=2$ [T, Corollary 3.7]. Assume that $d \geq 3$ and that our assertion holds true for $d-1$. Let $B=A /\left(a_{j}\right)$ with $1 \leq j \leq d$, and 
put $\bar{A}=B / \mathrm{H}_{\mathfrak{m}}^{0}(B)\left(=A / \mathrm{U}\left(a_{j}\right)\right)$. Then $\mathrm{H}_{\mathfrak{m}}^{i}(\bar{A}) \cong \mathrm{H}_{\mathfrak{m}}^{i}(B)$ for all $i \geq 1$. On the other hand, since $a_{j} \mathrm{H}_{\mathfrak{m}}^{i}(A)=(0)$ for $1 \leq i \leq d-1$ and $a_{j}$ is $A$-regular, we get for each $0 \leq i \leq d-2$ the short exact sequence

$$
0 \rightarrow \mathrm{H}_{\mathfrak{m}}^{i}(A) \rightarrow \mathrm{H}_{\mathfrak{m}}^{i}(B) \rightarrow \mathrm{H}_{\mathfrak{m}}^{i+1}(A) \rightarrow 0
$$

of local cohomology modules. Consequently, we get $\mathbb{I}(A)=\mathbb{I}(B)$ and

$$
\begin{aligned}
\mathrm{e}_{Q}^{1}(A)=\mathrm{e}_{Q B}^{1}(B) & =\mathrm{e}_{Q \bar{A}}^{1}(\bar{A}) \\
& \geq-\sum_{i=1}^{d-2}\left(\begin{array}{l}
d-3 \\
i-1
\end{array}\right) h^{i}(\bar{A}) \\
& =-\sum_{i=1}^{d-2}\left(\begin{array}{l}
d-3 \\
i-1
\end{array}\right) h^{i}(B) \\
& =-\sum_{i=1}^{d-2}\left(\begin{array}{l}
d-3 \\
i-1
\end{array}\right)\left[h^{i}(A)+h^{i+1}(A)\right] \\
& =-\sum_{i=1}^{d-1}\left(\begin{array}{l}
d-2 \\
i-1
\end{array}\right) h^{i}(A) \\
& =\mathrm{e}_{Q}^{1}(A)
\end{aligned}
$$

(see [GNi, Lemma 2.4]). Hence, the equality

$$
\mathrm{e}_{Q \bar{A}}^{1}(\bar{A})=-\sum_{i=1}^{d-2}\left(\begin{array}{c}
d-3 \\
i-1
\end{array}\right) h^{i}(\bar{A})
$$

holds true for the parameter ideal $Q \bar{A}$ in the generalized Cohen-Macaulay local ring $\bar{A}$. Thus the hypothesis of induction on $d$ yields that $Q \cdot\left[A / \mathrm{U}\left(a_{j}\right)\right]$ is a standard parameter ideal in $A / \mathrm{U}\left(a_{j}\right)$ for every $1 \leq j \leq d$. Therefore, $\mathrm{U}\left(a_{1}\right) \cap Q=\left(a_{1}\right)$ by Proposition 2.3 , so that $Q \cdot\left[A /\left(a_{1}\right)\right]$ is a standard parameter ideal in $A /\left(a_{1}\right)\left[\mathrm{T}\right.$, Corollary 2.3], since $Q \cdot\left[A / \mathrm{U}\left(a_{1}\right)\right]$ is a standard parameter ideal for $A / \mathrm{U}\left(a_{1}\right)$. Thus $Q$ is a standard parameter ideal [T, Corollary 2.4], since $\mathbb{I}(A)=\mathbb{I}\left(A /\left(a_{1}\right)\right)$.

We are now ready to prove Theorem 1.1. Let

$$
\Lambda(A)=\left\{\mathrm{e}_{Q}^{1}(A) \mid Q \text { is a parameter ideal in } A\right\} .
$$


Proof of Theorem 1.1. We have only to show the implication $(2) \Rightarrow(1)$. Since $\sharp \Lambda(A)=1$, by [GhGHOPV, Proposition 4.2], $A$ is a generalized CohenMacaulay local ring, so that $\Lambda(A)=\left\{-\sum_{i=1}^{d-1}\left(\begin{array}{c}d-2 \\ i-1\end{array}\right) h^{i}(A)\right\}$ by [Sch, Korollar 3.2]. Hence, by Theorem 2.1, every parameter ideal $Q$ is standard in $A$, because $\mathrm{e}_{Q}^{1}(A)=-\sum_{i=1}^{d-1}\left(\begin{array}{c}d-2 \\ i-1\end{array}\right) h^{i}(A)$, so that $A$ is a Buchsbaum local ring.

REMARK 2.4. One can arbitrarily control the values $h^{i}(A)=\ell_{A}\left(\mathrm{H}_{\mathfrak{m}}^{i}(A)\right)$ in Theorem 1.1. For example, let $d \geq 2$ and $h_{i} \geq 0(1 \leq i \leq d-1)$ be integers. Then, thanks to [G, Theorem 1.1], one gets a Buchsbaum local integral domain $(A, \mathfrak{m})$ with $\operatorname{dim} A=d$ and $\ell_{A}\left(\mathrm{H}_{\mathfrak{m}}^{i}(A)\right)=h_{i}$ for all $1 \leq i \leq d-1$. Therefore $A$ is unmixed and

$$
\Lambda(A)=\left\{-\sum_{i=1}^{d-1}\left(\begin{array}{l}
d-2 \\
i-1
\end{array}\right) h_{i}\right\}
$$

by [Sch, Korollar 3.2].

Unless $A$ is unmixed, Theorem 1.1 is no longer true, even if $\mathrm{e}_{Q}^{1}(A)=0$ for every parameter ideal $Q$ in $A$ (cf. [GhGHOPV, Theorem 2.1]). Let us note two examples.

EXAMPLE 2.5. Let $R$ be a regular local ring with the maximal ideal $\mathfrak{n}$ and $d=\operatorname{dim} R \geq 3$. Let $X_{1}, X_{2}, \ldots, X_{d}$ be a regular system of parameters of $R$. We put $\mathfrak{p}=\left(X_{1}, X_{2}, \ldots, X_{d-1}\right)$ and $D=R / \mathfrak{p}$. Then $D$ is a discrete valuation ring (DVR). Let $A=R \ltimes D$ denote the idealization of $D$ over $R$. Then $A$ is a Noetherian local ring with the maximal ideal $\mathfrak{m}=\mathfrak{n} \times D$ and $\operatorname{dim} A=d$. Let $Q$ be a parameter ideal in $A$, and put $\mathfrak{q}=\varphi(Q)$, where $\varphi: A \rightarrow R, \varphi(a, x)=a$ denotes the projection map. We then have

$$
\begin{aligned}
\ell_{A}\left(A / Q^{n+1}\right) & =\ell_{R}\left(R / \mathfrak{q}^{n+1}\right)+\ell_{D}\left(D / \mathfrak{q}^{n+1} D\right) \\
& =\ell_{R}(R / \mathfrak{q}) \cdot\left(\begin{array}{c}
n+d \\
d
\end{array}\right)+\ell_{D}(D / \mathfrak{q} D) \cdot\left(\begin{array}{c}
n+1 \\
1
\end{array}\right) \\
& =\mathrm{e}_{\mathfrak{q}}^{0}(R)\left(\begin{array}{c}
n+d \\
d
\end{array}\right)+\mathrm{e}_{\mathfrak{q} D}^{0}(D)\left(\begin{array}{c}
n+1 \\
1
\end{array}\right)
\end{aligned}
$$

for all integers $n \geq 0$, so that $\mathrm{e}_{Q}^{0}(A)=\mathrm{e}_{\mathfrak{q}}^{0}(R), \mathrm{e}_{Q}^{d-1}(A)=(-1)^{d-1} \mathrm{e}_{\mathfrak{q} D}^{0}(D)$, and $\mathrm{e}_{Q}^{i}(A)=0$ if $i \neq 0, d-1$. Hence, $\mathrm{e}_{Q}^{1}(A)$ is constant, but $A$ is not even a generalized Cohen-Macaulay local ring, because $\mathrm{H}_{\mathfrak{m}}^{1}(A)\left(\cong \mathrm{H}_{\mathfrak{n}}^{1}(D)\right)$ is not a finitely generated $A$-module. The local ring $A$ is not unmixed, although $\operatorname{depth} A=1$. 
EXAMPLE 2.6. Let $d \geq 3$ be an integer, and let $U=k\left[\left[X_{1}, X_{2}, \ldots, X_{d}, Y\right]\right]$ be the formal power series ring over a filed $k$. We look at the local ring

$$
A=U /\left[\left(X_{1}, X_{2}, \ldots, X_{d}\right) \cap(Y)\right] .
$$

Then $A$ is a reduced $\operatorname{ring}$ with $\operatorname{dim} A=d$. We put $R=U /(Y)$ and $D=$ $U /\left(X_{1}, X_{2}, \ldots, X_{d}\right)$. Let $Q$ be a parameter ideal in $A$. Then, since $D$ is a DVR and $R$ is a regular local ring with $\operatorname{dim} R=d$, thanks to the exact sequence $0 \rightarrow D \rightarrow A \rightarrow R \rightarrow 0$, similarly as in Example 2.5 we get that $\operatorname{depth} A=1$ and that

$$
\begin{aligned}
\ell_{A}\left(A / Q^{n+1}\right) & =\ell_{R}\left(R / Q^{n+1} R\right)+\ell_{D}\left(D / Q^{n+1} D\right) \\
& =\ell_{R}(R / Q R) \cdot\left(\begin{array}{c}
n+d \\
d
\end{array}\right)+\ell_{D}(D / Q D) \cdot\left(\begin{array}{c}
n+1 \\
1
\end{array}\right) \\
& =\mathrm{e}_{Q R}^{0}(R)\left(\begin{array}{c}
n+d \\
d
\end{array}\right)+\mathrm{e}_{Q D}^{0}(D)\left(\begin{array}{c}
n+1 \\
1
\end{array}\right)
\end{aligned}
$$

for all integers $n \geq 0$. Therefore $\mathrm{e}_{Q}^{1}(A)=0$ for every parameter ideal $Q$ in $A$. However, $A$ is not a Buchsbaum ring, since $\mathrm{H}_{\mathfrak{m}}^{1}(A)=\mathrm{H}_{\mathfrak{m}}^{1}(D)$ is not a finitely generated $A$-module, where $\mathfrak{m}$ denotes the maximal ideal in $A$.

\section{$\S 3$. Characterization of local rings with constant $\mathrm{e}_{Q}^{1}(A)$}

We close this paper with a characterization of Noetherian local rings $A$ possessing $\sharp \Lambda(A)=1$. Let us note the following.

Proposition 3.1 ([GhGHOPV, Proposition 4.7]). Suppose that $d=$ $\operatorname{dim} A \geq 2$, and let $U$ be the unmixed component of the ideal (0) in $A$. Assume that there exists an integer $t \geq 0$ such that $\mathrm{e}_{Q}^{1}(A)=-t$ for every parameter ideal $Q$ in $A$. Then $\operatorname{dim}_{A} U \leq d-2$ and $\mathrm{e}_{\mathfrak{q}}^{1}(A / U)=-t$ for every parameter ideal $\mathfrak{q}$ in $A / U$.

The goal of this paper is the following.

TheOREM 3.2. Suppose that $d=\operatorname{dim} A \geq 2$. Then the following two conditions are equivalent.

(1) $\sharp \Lambda(A)=1$.

(2) Let $U=\mathrm{U}_{\widehat{A}}(0)$ be the unmixed component of the ideal (0) in the $\mathfrak{m}$-adic completion $\widehat{A}$ of $A$. Then $\operatorname{dim}_{\widehat{A}} U \leq d-2$, and $\widehat{A} / U$ is a Buchsbaum local ring. 
When this is the case, one has the equality $\mathrm{e}_{Q}^{1}(A)=-\sum_{i=1}^{d-1}\left(\begin{array}{c}d-2 \\ i-1\end{array}\right) h^{i}(\widehat{A} / U)$ for every parameter ideal $Q$ in $A$.

Proof. (1) $\Rightarrow(2)$. For every parameter ideal $\mathfrak{q}$ of $\widehat{A}$, we have $\mathfrak{q}=(\mathfrak{q} \cap$ $A) \widehat{A}$, so that $\mathfrak{q} \cap A$ is a parameter ideal in $A$. Hence, $\Lambda(\widehat{A})=\Lambda(A)$, so the implication follows from Theorem 1.1 and Proposition 3.1.

$(2) \Rightarrow(1)$. Since $\operatorname{dim}_{\widehat{A}} U \leq d-2$ and $\widehat{A} / U$ is a Buchsbaum local ring, we get $\# \Lambda(\widehat{A})=1$ by $[$ GhGHOPV, Lemma $2.4(\mathrm{c})]$. Therefore $\# \Lambda(A)=1$.

\section{REFERENCES}

[GhGHOPV] L. Ghezzi, S. Goto, J. Hong, K. Ozeki, T. T. Phuong, and W. V. Vasconcelos, Cohen-Macaulayness versus the vanishing of the first Hilbert coefficient of parameter ideals, J. London Math. Soc. (2) 81 (2010), 679-695.

[GhHV] L. Ghezzi, J.-Y. Hong, and W. V. Vasconcelos, The signature of the Chern coefficients of local rings, Math. Res. Lett. 16 (2009), 279-289.

[G] S. Goto, On Buchsbaum rings, J. Algebra 67 (1980), 272-279.

[GNi] S. Goto and K. Nishida, Hilbert coefficients and Buchsbaumness of associated graded rings, J. Pure Appl. Algebra 181 (2003), 61-74.

$[\mathrm{H}] \quad$ C. Huneke, The theory of d-sequences and powers of ideals, Adv. in Math. 46 (1982), 249-279.

[MV] M. Mandal and J. K. Verma, On the Chern number of an ideal, Proc. Amer. Math. Soc. 138 (2010), 1995-1999.

[Sch] P. Schenzel, Multiplizitäten in verallgemeinerten Cohen-Macaulay-Moduln, Math. Nachr. 88 (1979), 295-306.

[STC] P. Schenzel, N. V. Trung, and N. T. Cuong, Verallgemeinerte CohenMacaulay-Moduln, Math. Nachr. 85 (1978), 57-73.

[SV] J. Stückrad and W. Vogel, Buchsbaum Rings and Applications, Springer, Berlin, 1986.

[T] N. V. Trung, Toward a theory of generalized Cohen-Macaulay modules, Nagoya Math. J. 102 (1986), 1-49.

[V] W. V. Vasconcelos, The Chern coefficients of local rings, Mich. Math. J. 57 (2008), 725-743.

Shiro Goto

Department of Mathematics

School of Science and Technology

Meiji University

Kawasaki 214-8571

Japan

goto@math.meiji.ac.jp 
Kazuho Ozeki

Department of Mathematics

School of Science and Technology

Meiji University

Kawasaki 214-8571

Japan

kozeki@math.meiji.ac.jp 